\title{
命HAD
}

ISSN-L: 2530-5115

(c) (1)(2)

DOI: http://doi.org/10.22585/hospdomic.v4i1.90

\section{Jornada Nacional de Hospitalización Domiciliaria en Chile}

II National Meeting of Hospital at Home in Chile

M. Fernanda González-Cuesta', Francisco Freire-Figueroa'

1. Complejo Asistencial Dr. Sótero del Río, Unidad de Hospitalización Domiciliaria, Santiago, Chile.

Correspondencia/Correspondence

M. Fernanda González Cuesta

fe.gonzalezcuesta@gmail.com

Francisco Freire Figueroa

f.freire.figueroa@gmail.com

Recibido/Received

18.10.2019

Aceptado/Accepted

17.12.2020
Conflicto de Intereses/Competing interest

La autora y el autor del trabajo declaran no tener ningún conflicto de interés

CÓMO CITAR ESTE TRABAJO | HOW TO CITE THIS PAPER

González-Cuesta MF, Freire-Figueroa F. II Jornada Nacional de Hospitalización Domiciliaria en Chile. Hosp Domic. 2020;4(1):9-13. 


\section{RESUMEN}

Se presenta brevemente la II Jornada Nacional de Hospitalización Domiciliaria, organizada por la Unidad de Hospitalización Domiciliaria del Complejo Asistencial Dr. Sótero del Río en Santiago de Chile, los días 10 y 11 de Octubre del presente año. El objetivo de esta Jornada se fundamenta en el esfuerzo por contribuir a la formación y crecimiento profesional de todos los actores sanitarios que desempeñan sus funciones laborales en Unidades de Hospitalización Domiciliaria, pertenecientes a la red de salud pública de Chile. Además, existe el intento vertebrador de ampliar sus objetivos a otros países del ámbito iberoamericano y también, contribuir con la elaboración y difusión de la investigación científica.

\section{ABSTRACT}

We briefly present the II National Meeting of Hospital at Home, organized by the Hospital at Home Unit of the Dr. Sótero del Río Hospital Complex in Santiago of Chile, on October 10 and 11 of this year. The goal of this Meeting underlies on the effort of contribute to the training and professional growth of all health actors, who perform their duties in Hospital at Homes Units, that belong to the public health network of Chile. In addition, there's a backbone attempt to broaden its objectives to other countries in the Latin American area and also, contribute to the development and dissemination of scientific research. 
Una gran convocatoria recibió la II Jornada Nacional de Hospitalización Domiciliaria, organizada por la Unidad de Hospitalización Domiciliaria del Complejo Asistencial Dr. Sótero del Río en Santiago de Chile, los días 10 y 11 de Octubre del presente año (ver figura 1).

Figura 1

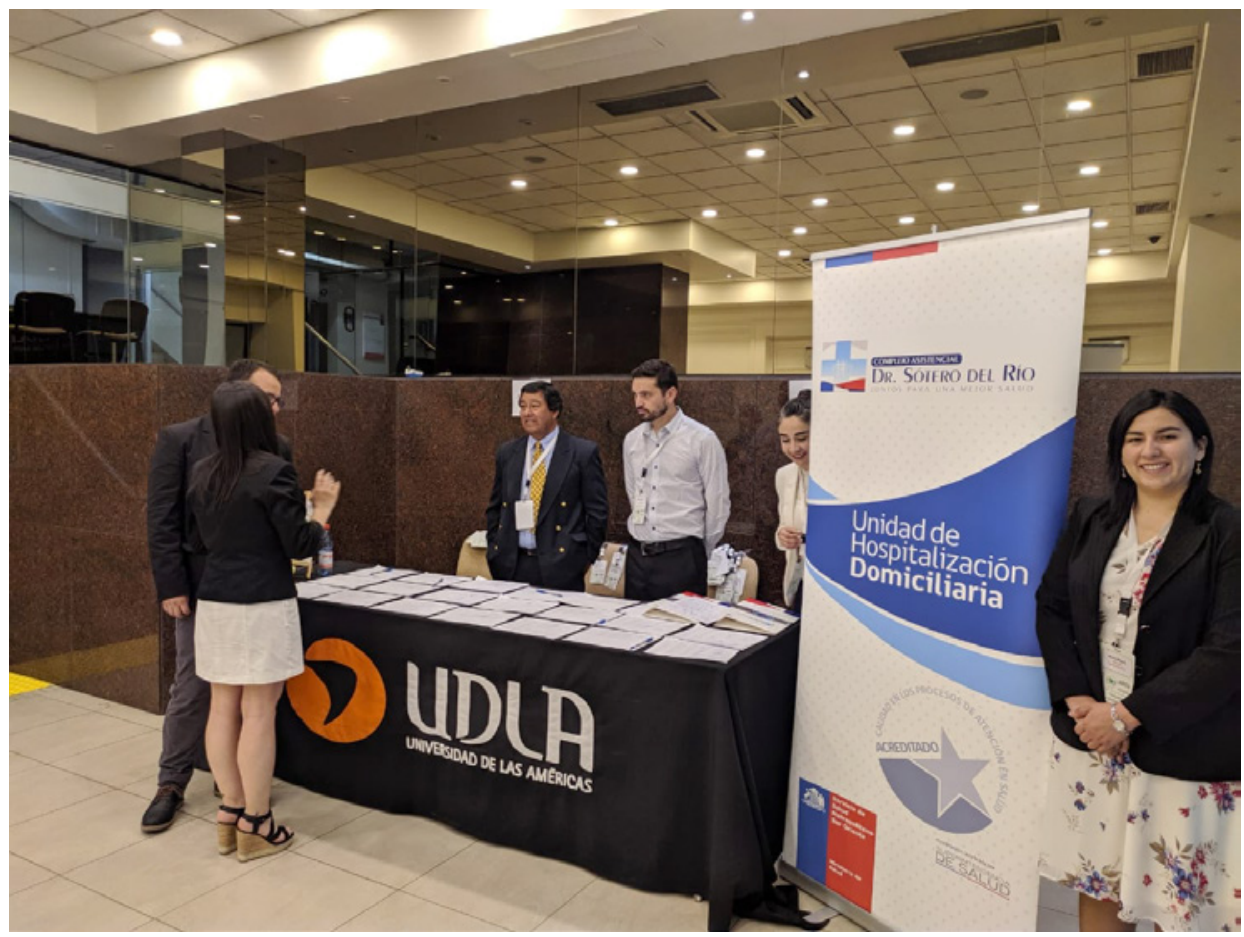

Esta instancia se orienta como un constante esfuerzo por contribuir a la formación y crecimiento profesional de todos los actores sanitarios que desempeñan sus funciones laborales en Unidades de Hospitalización Domiciliaria, pertenecientes a la red de salud pública de nuestro país.

El encuentro contó con la presencia de más de doscientas personas asistentes, dentro de los que destacan diversos profesionales médicos y no médicos, técnicos de nivel superior, docentes, estudiantes y administrativos. Respondieron a la convocatoria cuarenta y un establecimientos hospitalarios de veinticinco localidades del país. Participaron asimismo, algunas autoridades y representantes del Ministerio de Salud, Servicios de Salud y del Complejo Hospitalario organizador.

Diez y seis fueron los ponentes encargados de desarrollar los diferentes contenidos durante el evento (ver figura 2). De ellos, dos fueron invitados extranjeros desde Argentina y España, quienes en modalidad presencial y videograbación respectivamente, compartieron la realidad local y desafíos de sus correspondientes servicios de Hospitalización Domiciliaria (HD). Además de los expositores ya mencionados, se contó con la participación de seis grupos en la modalidad de presentación de trabajos libres, circunstancia que tiene como objetivo fomentar la investigación en Hospitalización Domiciliaria a nivel nacional. Además, dentro de las actividades del encuentro se presentó la revista Hospital a Domicilio (ver figura 3) y se informó ampliamente, a todos los asisten- 
tes, del carácter multidisciplinar de la publicación y su enfoque hacia los profesionales relacionados con la prestación de servicios de salud, en el domicilio, dependientes del hospital.

Figura 2

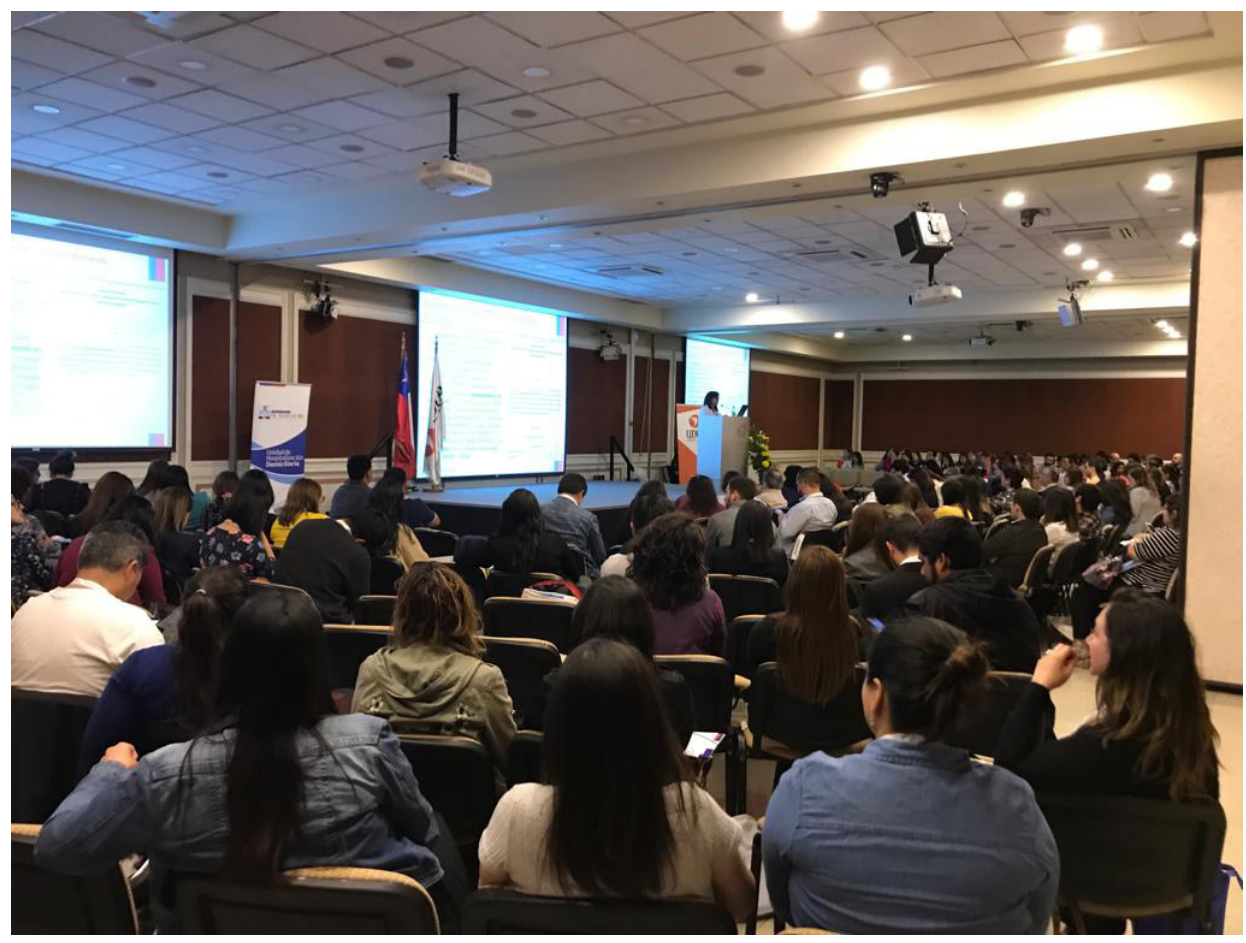

Las temáticas desarrolladas se vinculan al área clínica, social, de gestión y de investigación. Destacando innovadores proyectos tales como el seguimiento de pacientes Prematuros Extremos para la prevención de consultas en Servicios de Atención de Urgencia, la utilización de Catéteres Venosos Centrales de Inserción Periférica para la administración segura de tratamiento endovenoso prolongado, el establecimiento de Indicadores para evaluar la Calidad de Salud en HD, presentación de la norma técnica desde el Ministerio de Salud y el cuidado de la Espiritualidad dentro del equipo interdisciplinario que atiende usuarios en condición de fin de vida.

Con respecto a la evaluación, se han destacado positivamente la organización, los contenidos, la convocatoria y la infraestructura del lugar. Como oportunidad de mejora se ha planteado realizar una de las próximas versiones fuera de la capital, para permitir la mayor participación desde otras regiones del país.

Como parte del equipo organizador, esperamos poder seguir entregando esta instancia de encuentro y aprendizaje en futuras versiones. Nos quedamos con el desafío de cumplir las altas expectativas que nuestras autoridades y asistentes nos han manifestado de manera verbal y por escrito. 


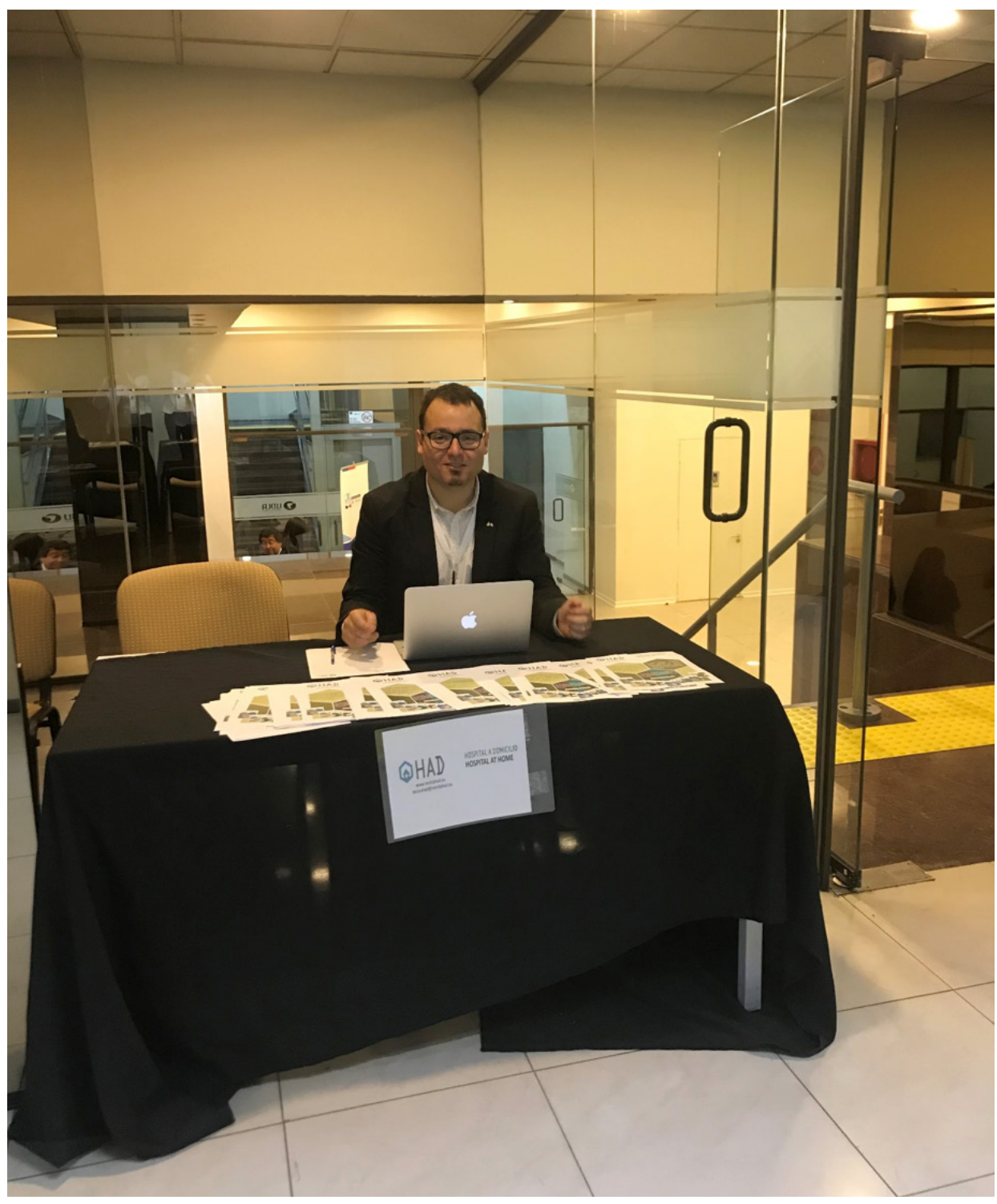

